\title{
Gastrointestinal disasters of cetuximab in the treatment of metastatic colorectal cancer: Mechanism and its effect on prognosis
}

\author{
Yue $\mathrm{Wu}^{\mathrm{a}}$, Haowei Jia ${ }^{\mathrm{a}}$, Xinqi Bao ${ }^{\mathrm{a}}$, Tianyu Zhu ${ }^{\mathrm{a}}$, Ruixin $\mathrm{Li}^{\mathrm{a}}$, Hongchao Zhao ${ }^{\mathrm{a}}$, Junfeng Sun ${ }^{\mathrm{a},{ }^{*}}$ \\ ${ }^{a}$ Department of Gastrointestinal Surgery, The First Affiliated Hospital of Zhengzhou University, Zhengzhou, China.
}

\begin{abstract}
Colorectal cancer (CRC) is among the top three cancers worldwide in terms of incidence and associated mortality. Colorectal cancer (CRC) is responsible for more than 880,000 deaths annually. The number of of CRC cases worldwide continues to increase, posing a serious threat to human health. Surgery and chemotherapy are the first treatments for CRC patients. The majority of CRC patients are diagnosed at an advanced stage, as symptoms are usually not apparent and difficult to diagnose in the early stage. The prognosis of metastatic CRC (mCRC) has long been unsatisfactory. Targeted drugs therapy, which targeting at specific genes and proteins, is a new treatment approach to CRC. Cetuximab is one of the most widely studied targeted drugs. By competitively binding to the epithelial growth factor receptor (EGFR), cetuximab inhibits the EGF and binding of the EGF ligand to the EGFR, thereby inhibiting tumor cell growth, invasion, and metastasis and inducing tumor cell apoptosis. The curative effect of cetuximab as a treatment for many kinds of tumors, especially mCRC, has been confirmed. Cetuximab combined with chemotherapy or monotherapy is used as first-line treatment in patients with RAS(rat sarcoma,Ras) wild-type mCRC. However, adverse drug reactions (ADRs) associated with the clinical application of cetuximab are attracting increasing attention, with numerous studies reporting adverse effects of cetuximab on the gastrointestinal system, with these effects having adverse consequences for the prognosis of CRC. In this review, we focus mainly on gastrointestinal disasters on cetuximab treatment for mCRC from three areas: the intestinal mucosal barrier (IMB), gut microbiota (GM)-host immune balance, and bacterial metabolites short chain fatty acids (SCFAs), and the impact of these effects on the prognosis of mCRC. We also make suggestions aimed at aiding oncological understanding of cetuximab as a treatment for mCRC.
\end{abstract}

Keywords: Bacterial metabolites, cetuximab, colorectal cancer, gut microbiota-host immune balance, gastrointestinal disasters

\section{Introduction}

Cetuximab is an immunoglobulin G1(IgG1)human/mouse chimeric monoclonal antibody targeting the extracellular region of the EGFR [1].It can specifically bind to EGFRrelated domains on the surface of a variety of cancer cells, competitively block the corresponding ligands, and inhibit the activation of RAS-RAF. RAS-RAF can induce the

\footnotetext{
* Corresponding author: Junfeng Sun

Mailing address: Department of Gastrointestinal Surgery, The First Affiliated Hospital of Zhengzhou University, Zhengzhou, China.

E-mail: sunjunfenger@126.com
}

Received: 12 May 2020 / Accepted: 08 June 2020 phosphorylation and activation of receptor-related kinases (MAPK, MEK, and ERK) and regulate the expression of transcription factors [2]. Cetuximab can also activate $\mathrm{PI} 3 \mathrm{~K}$ and affect the $\mathrm{SH} 3$ domain of AKT, thereby regulating cell growth and apoptosis. Moreover, by inhibiting the activation of PLC- $\gamma 1$ through the EGFR, EGFR inhibitors affect cell movement, growth, and differentiation.This can lead to membrane wrinkling, which is critical for the proliferation and apoptosis of cancer cells [3]. In addition to inhibiting EGFRs, cetuximab exerts antitumor effects in many other ways, such as inhibiting the production of vascular endothelial growth factor (VEGF), inducing natural killer cells (NK cells) to kill tumor cells through antibody-dependent cell mediated cytotoxicity, regulating hypoxia factor 1-a and Bcl-2 proto oncogenes, activating the autophagy genes BECLIN1 and HVPS34, and inducing the autophagy of tumor cells [4]. Thus, cetuximab plays an antitumor role at multiple levels and paths. 


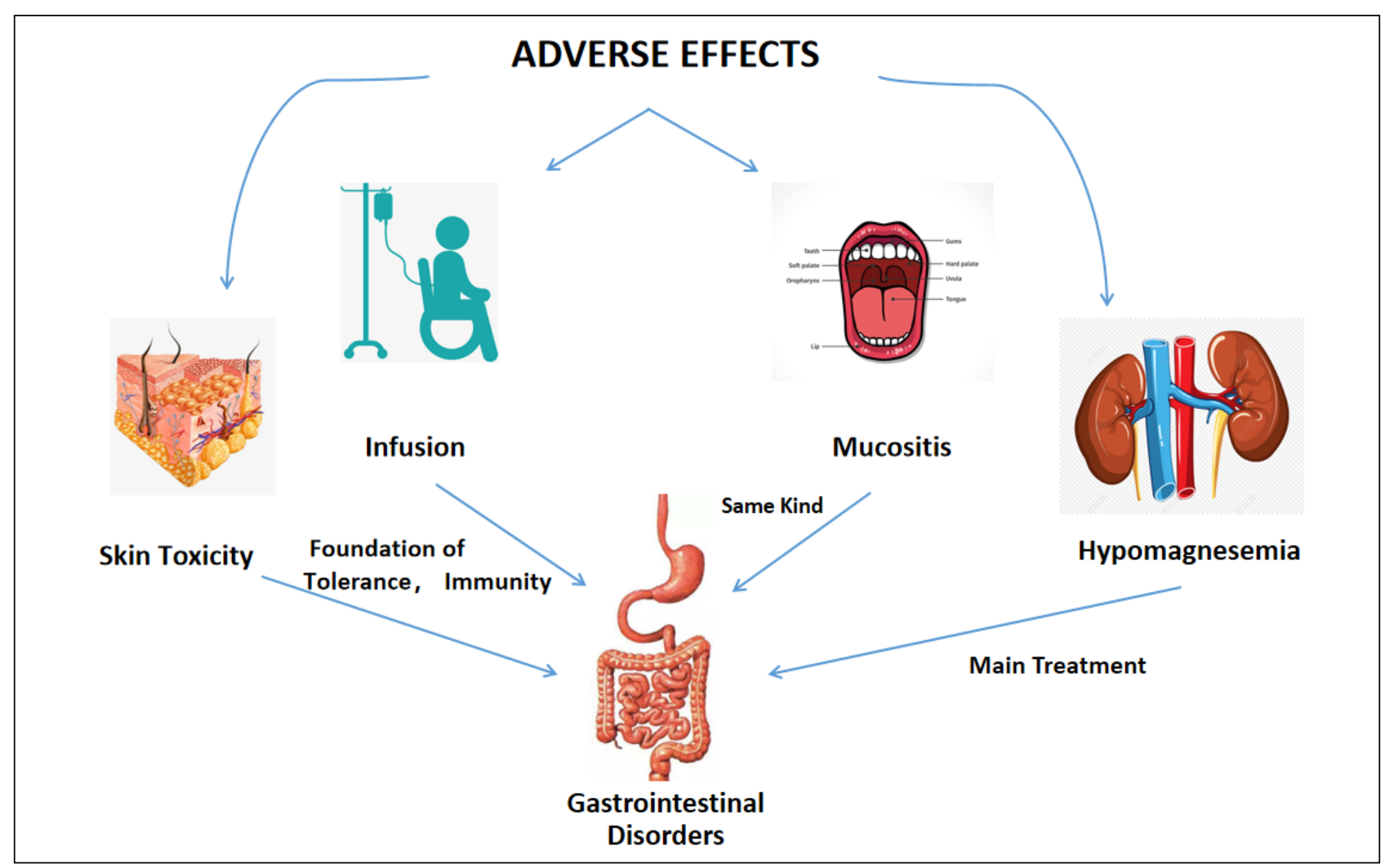

Figure 1. The role of gastrointestinal disorders in major adverse drug reactions.

Phase III clinical trials showed that combination treatment with cetuximab and irinotecan [5], FOLFOX [6], or multitarget drugs [7-9] was more effective in mCRC treatment than cetuximab treatment alone. The National Comprehensive Cancer Network and Food and Drug Administration have recommended that cetuximab combined with classical chemotherapy can be used as a first-line treatment in patients with RAS wild-type mCRC [10]. Notably, not all RAS wild-type CRC patients are sensitive to cetuximab, with studies showing that mutations of the RAS gene (NRAS and KRAS) [11] and V600E BRAF [12] caused drug resistance. In previous research, a PI3K mutation and PTEN loss affected the efficacy of cetuximab combined with chemotherapy in mCRC treatment [13].

In terms of adverse drug reactions (ADRs), skin reactions, hypomagnesemia, mucositis, and infusion-related reactions have attracted much attention [14]. Gastrointestinal disasters (GDs) refer to the expression of mucositis in the intestinal mucosa [15]. We find GDs play significant roles in all ADRs (Figure 1) and relate to the occurrence, treatment, and tolerance. In addition, GDs affect the efficacy of cetuximab in many ways and have a profound impact on the prognosis of CRC.

Previous reviews focused on the mechanism of action and resistance of cetuximab [16,17]. We review mainly adverse drug reactions (ADRs) of cetuximab when administered as monotherapy or in combination with classical chemotherapy, focusing on the mechanism underlying its effects on the gastrointestinal system and its influence on the prognosis of $\mathrm{mCRC}$. We review clinical studies of ce- tuximab in combination with chemotherapy, radiotherapy, and multitarget drugs combinations.

\section{ADRs of Cetuximab}

Cetuximab is generally well tolerated. However, with the clinical application of cetuximab, its adverse drug reactions (ADRs) are attracting increasing attention. The main ADRs include skin reactions, hypomagnesemia, mucositis, and transfusion-related reactions [18]. It should be noted that GDs jump to the most important ADRs after the application of cetuximab combined with chemotherapy [19]. According to the Food and Drug Administration Adverse Event Reporting System and the EudraVigilance database, the majority of ADRs of cetuximab affect the gastrointestinal system (Figure 2). However, in most cases, these skin reactions are mild or moderate rashes [20]. Based on our review of clinical studies, the incidence of severe rashes associated with cetuximab is low [21]. Infusionrelated reactions may recur, they can be effectively controlled by conventional antihistamines and corticosteroids [22]. Although the digestive tract is not directly related to skin reactions and infusion reactions, its condition is closely related to the nutrition and immune status of the patient, which is the basis of ADR tolerance [23]. The EGF regulates the activity and distribution of TRPM6 and mutations in the EGFR gene .Cetuximab acts on EGFRs expressing a lot in the kidney, resulting in hypomagnesemia. Controlling GDs, especially diarrhea, is the first step 


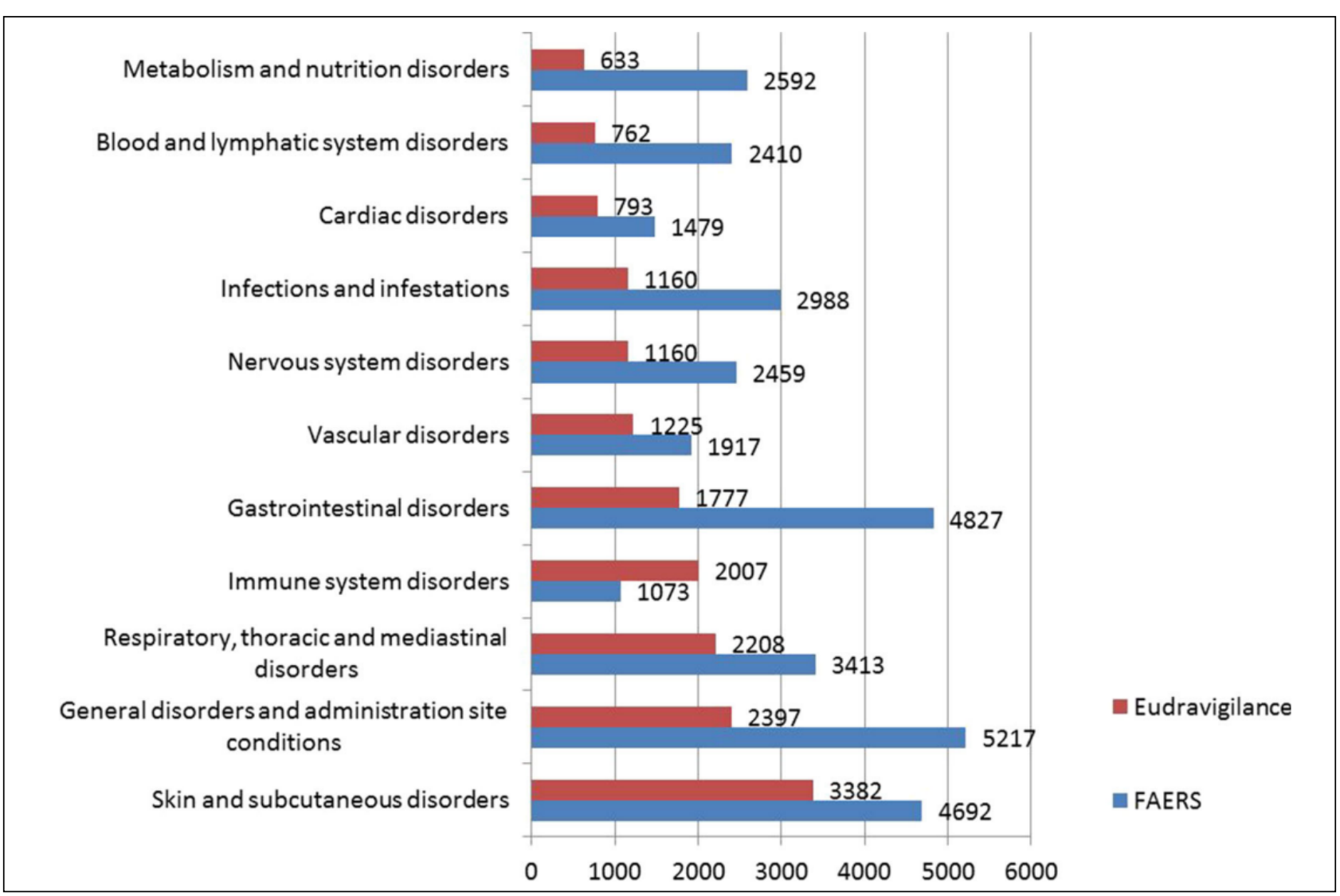

Figure 2. Numbers of cases of ADRs reported in EudraVigilance and FEARS (cited from Fornasier, G) [18].

in alleviating hypomagnesemia [24]. In terms of mucositis, most reports have focused on oral mucosal lesions [25]. However, mucosa are widely distributed in the digestive tract, where EGFRs are widely expressed. Thus, GDs are symptoms of mucositis in the digestive tract. Several studies have confirmed a direct causal relation between gastrointestinal disorders(GDs) and intestinal mucositis [26,27]. Based on the discussion above, it can be concluded that ADRs of cetuximab include GDs.

To sum up, Many studies have found that ADRs of antitumor drugs, including cetuximab, occur through multiple channels and that these ADRs, affect the antitumor effect of the drugs, tumoral progression, and the prognosis of CRC [28]. In this review, we focus on the impacts of ADRs of cetuximab on the intestinal mucosal barrier (IMB), gut microbiota (GM)-host immune balance, and microbial metabolites short chain fatty acids (SCFAs) (Figure 3).

\section{IMB induces GDs and Contributes to the Tumor Growth Microenvironment}

The destruction of the IMB plays a vital role in inducing mucositis [29]. Mucositis in the gastrointestinal tract is a major ADR of cetuximab and the most common ADR of cetuximab when it is combined with irinotecan and FOLFOX $[6,30]$. The main mechanism underlying mucositis induced by cetuximab of EGFR inhibition as epidermal growth factor receptors (EGFRs) are widely distributed in the digestive trace, and $25-77 \%$ of CRC cases overexpress EGFRs [31]. According to our review, EGFR inhibitors affect almost all the major components of the IMB. The IMB can broadly be divided into a physical barrier and chemical barrier [32]. The physical barrier consists of four types of intestinal epithelial cells: absorptive intestinal cells, goblet cells producing mucin, Paneth cells producing antimicrobial peptides (AMPs), and endocrine cells produced by hormones. The chemical barrier mainly consists of a mucus layer containing mucin and AMPs and secretory immunoglobulin A ( $\operatorname{sIgA}$ ) [33]. Mucin is the skeleton of the mucus layer and works as isolation. IgA and AMPs can kill GM directly and work with mucin to isolate bacteria from intestinal epithelial cells, including symbionts [34]. Yasuda-Onozawa et al. found that the EGFR/Akt serine/threonine kinase 1 pathway induced the expression of mucin 2 and oligomeric mucus/gel forming mRNA and promoted the production of mucin in goblet cells [35]. EGFR inhibitors reduce the production of mucin, and the integrity of the IMB is difficult to maintain when mucin production is reduced. Gut microbiota (GM) and other components in the gut directly break through the gap of the IMB and come into contact with intestinal epithelial cells, resulting in mucosal inflammation [36].

This may be the initial mechanism of EGFR inhibition, leading to GDs and directly or indirectly inducing 


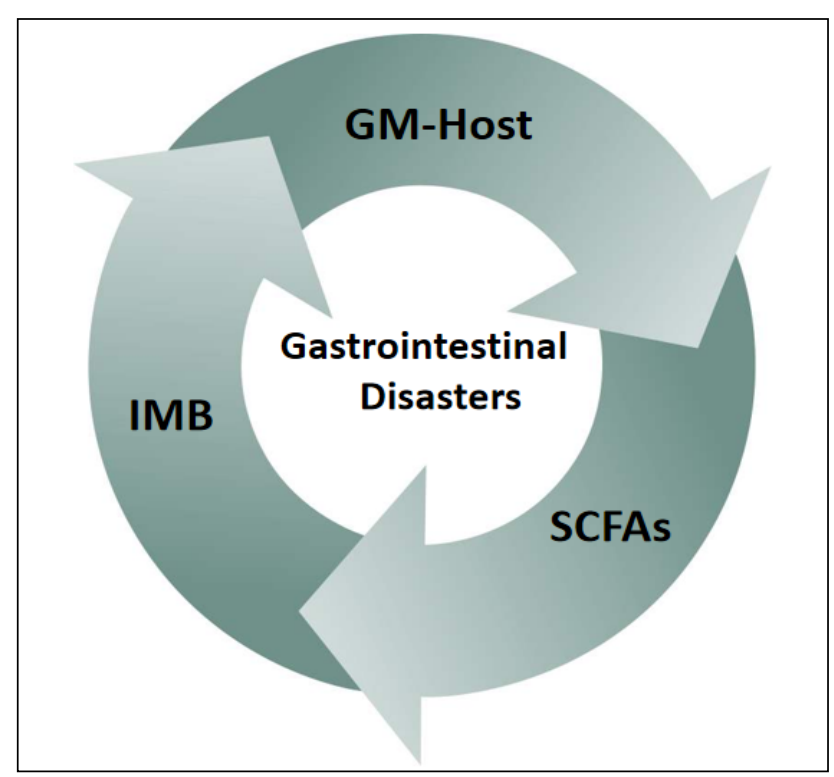

Figure 3. Interactions between the intestinal mucosal barrier (IMB), gut microbiota (GM)-host immune balance, and short chain fatty acids (SCFAs) leading to GDs.

multiple domino effects. Previous research showed that butyrate, a bacterial metabolite, increased mucin secretion [37]. If GMs are disordered by drugs and the inflammatory environment, butyrate will decrease significantly, and then, mucin production decrease with it. ErbB 3 is a member of the EGFR family, which inhibits Atoh 1 levels, mediated by PI3K, to limit the number of Paneth cells and AMPs will reduce after that [38]. Meanwhile, dendritic cells (DCs) are activated in the inflammatory state and induce B cells to produce IgA [39]. Although AMPs and IgA increase in response to EGFR inhibitors and kill invasive GM, they do not act as a physical barrier. In addition, a suitable reaction place for AMPs and IgA decrease due to mucin deficiency [40]. Thus, increasing the concentration of AMP and IgA cannot completely prevent bacteria from contacting with the IMB intestinal epithelial cells. On the contrary, Extensive GM mortality results in a dramatic reduction in butyrate production and an associated reduction in mucin. In addition, previous research showed that inflammation led to high permeability in and between epithelial cells and diarrhea exacerbates the loss of active ingredients of the IMB [41,42].

In mCRC, the status of the IMB is closely related to intestinal inflammation which is associated with the tumor growth environment and prognosis of CRC patients [43]. Since 1863, inflammation has been recognized as a high risk factor for cancer with less than $10 \%$ of cancers caused by gene mutations, and more than $20 \%$ related to microbial infections [44]. Chronic inflammation is a recognized risk factor for $\mathrm{CRC}$, and most patients with $\mathrm{mCRC}$ have chronic inflammation [45]. Intestinal flora disturbance caused by drugs and tumor rejection aggravate the original intestinal inflammation [46]. Following the destruction of the IMB, the immune response and inflammatory response induce the proliferation and differentiation of a variety of immune cells, which produce a large number of cytokines, forming a microenvironment for tumor growth that facilitates the occurrence, maintenance, and development of tumors [47]. Therefore, the destruction of the IMB caused by EGFR inhibitors initiates mucositis in the gut and not only contributes to ADRs but also antagonizes the curative effect of antitumor drugs, thereby having a profound impact on the prognosis.

\section{The Destruction of the GM-Host Immune Bal- ance Affects the Prognosis of $\mathrm{mCRC}$}

The immune balance between GM and the host is the result of coevolution [48]. The host provides a stable environment for GM, which have a wide range of functions, affecting the occurrence and development of various diseases, such as inflammatory bowel disease(IBD) and CRC [49]. The gut immune system must maintain a delicate balance between tolerance and immunity. It is found that this effect is realized by butyrate. Butyrate, a short chain fatty acid (SCFA), is the main metabolite of intestinal bacteria [50]. Butyrate can inhibit the differentiation of bone marrow stem cells into DCs, thereby maintaining host immunoreactivity at a low level [51]. Drugs, especially antibiotics and antitumor drugs, can lead to the destruction of the GM-host immune balance, with greater effects than either diet or inflammation [52]. A previous study showed that cetuximab combined with XELOX did not significantly improve overall survival and progression-free survival (PFS) of patients with mCRC as compared with XELOX alone [53].These suggest that the intestinal environment influence the drug efficacy.In the following, we will illustrate this effect from two aspects: host and GMs.

In terms of the host, a variety of host immune cells are activated by inflammation and the immune response, among which the proliferation and differentiation of DCs upregulate a proportion of $\mathrm{B}$ cells and $\mathrm{T}$ (Th1, Th2, Th17, and Treg) cells [54], leading to chemotherapy-induced enteritis and other side effects. Various growth factors, reactive oxygen species, and nitrogen produced by inflammatory cells persisting damage DNAs under and even after the inflammatory state. As a result, DNAs damaging give rise to gene mutations and the potential development of cancers [55].

Under pathological conditions, the proliferation and differentiation of DCs induced by intestinal bacteria due to translocation of bacteria across the IMB, allowing the bacteria to come into contact with intestinal epithelial cells [56]. In contrast, under normal conditions, Metabolites, such as butyrate, can pass the IMB and contact with intestinal epithelial cells easily [57].These metabolites will inhibits DCs proliferation and differentiation, but bacterials face many difficulties in front of IMB (Figure 4). Panebianco [58] confirmed that the immune and inflammation effect causing by bacterias was not realized by its translocation but metabolite. The latter explains why the host immune system can be activated, as well as inhibited, by GM.

Specifically, GM include bacteria, fungi, archaea, viruses 


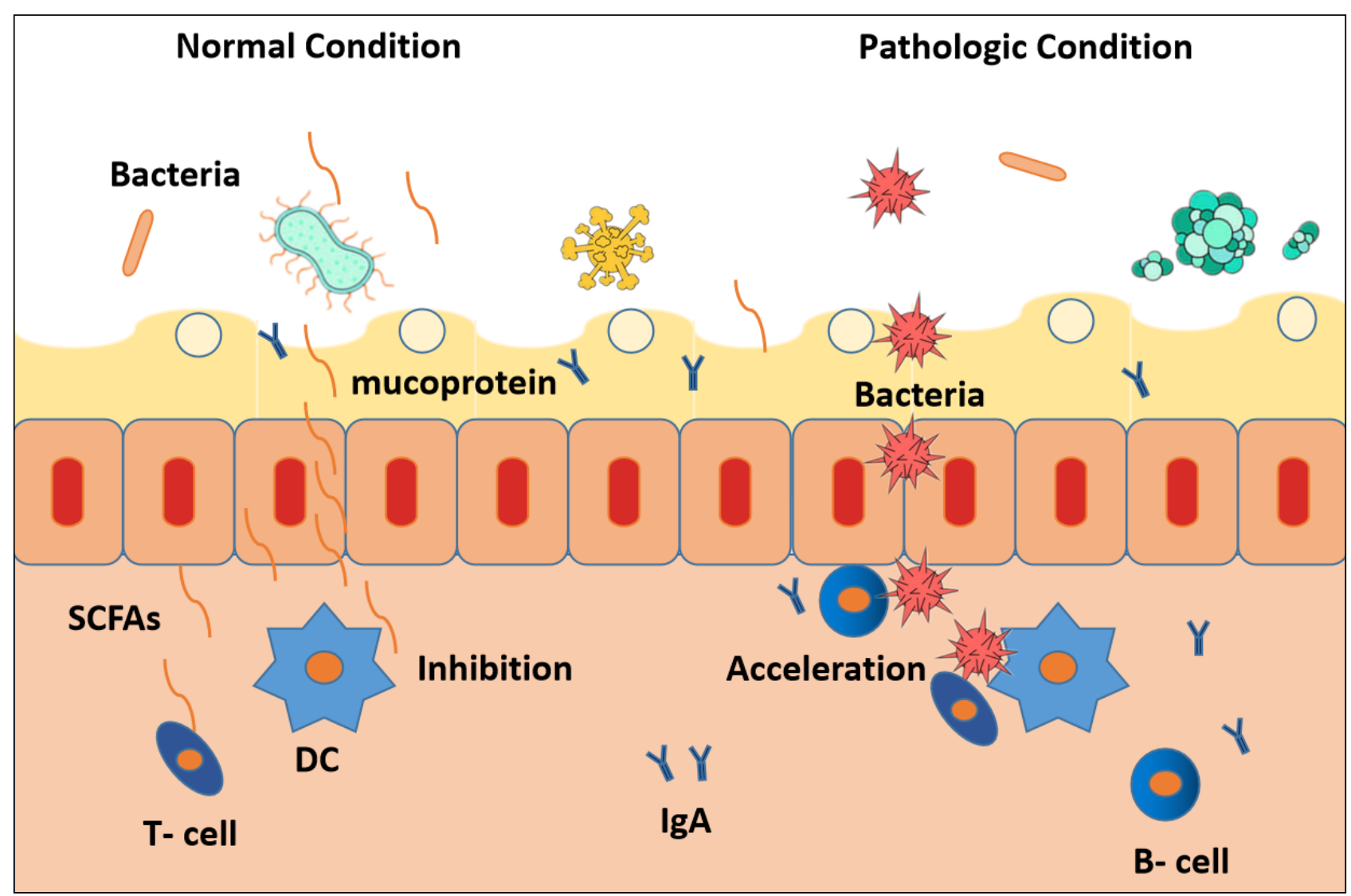

Figure 4. The action mode of IBM, GT-Host, SCFAs working under normal condition versus pathologic condition.

and parasites [59]. We're concerned about the effects of bacteria on the host and disease because bacteria are dominant in GM.GM disorders caused by drugs are mainly manifested by a decrease in the quantity and type [60]. In patients treated with irinotecan and FOLFOX, the number and species of intestinal bacteria all decrease shown by $16 \mathrm{~S}$ rRNA gene detection [61]. In an epidemiological investigation, a decrease in GM was associated with an increase in CRC morbidity [62]. Another study found that bacteria favored mucin as a carbon source in the absence of dietary fiber, which further aggravated IMB damage, increased bacterial contact with intestinal epithelial cells, and promoted the formation of a tumor microenvironment in the host gut [63]. However, whether this phenomenon existsin CRC patients treated with antibiotics and chemotherapy drugs has not been studied. Therefore, it can be concluded the destruction of the IMB by EGFR inhibitors leads to the destruction of the GM-host immune balance. The latter is a vital mechanism underlying gastrointestinal disorders, drug efficacy, and prognosis of mCRC.

\section{SCFAs Have Anti-Inflammatory and Antitumor Effects}

It can be seen from the above discussion that the integrity of the IMB and the immune balance between GM and the host are interdependent and interacted. SCFAs are running with them working as important media and signal [64]. SCFAs, metabolites of bacteria degraded from dietary fiber, include acetate, propionate, and butyrate. GMs exert an antitumor effect mainly through their metabolites [65]. Histone deacetylase inhibitors are widely used in cancer treatment [66]. Both propionate and butyrate inhibit histone deacetylase activity [67]. Propionate and butyrate decrease because of bacteria are damaged by antitumor drugs. As a result, the antitumor power of the host itself is clipped. According to some studies, the SCFA acetate plays a protective signal molecule acting on $\mathrm{G}$ proteincoupled receptor 109A(GPR109A) [68] and GPR43/41 [69] of host cells to regulate their energy metabolis.

In CRC, tumor location is an important factor in determining the reactivity of cetuximab [70]. Colorectal cancer (CRC) is usually classified as left or right sided, depending on the location of the tumor or tumors. In left-sided $\mathrm{CRC}$, the tumors originate in the flexure of the spleen, descending colon, and sigmoid colon, whereas $\mathrm{n}$ rightsided $\mathrm{CRC}$, the tumors originate in the cecum, ascending colon, flexure of the liver, and transverse colon [71]. As shown by multiple studies, EGFR inhibitors(such as cetuximab) are superior to left colon cancer with RAS wildtype comparing with the right, and it is recommended that patients with left RAS wild-type colon cancer should be given EGFR inhibitors [72,73]. Interestingly, SCFAs show a remarkable distribution and absorption gradient [74]. SCFAs are well absorbed in the distal colon but not fully 
absorbed in the proximal colon. According to Cummings et al et al., the transport effect of the sodium-coupled monocarboxylate transporter SLC5A8 and H-coupled low-affinity monocarboxylate transporter SLC16A makes the absorption efficiency of SCFAs greater in the distal colon than that in the proximal [75]. SCFAs play a biological role on the host only after they are absorbed [76]. As SCFAs have been shown to be effective as intestinal mucosal protective agents and to have antitumor functions, the absorption efficiency of SCFAs potentially explain the efficacy of cetuximab in CRC patients. However, the reason for the difference in the positional sensitivity of cetuximab in the colon is not clear, and research is lacking on the specific mechanism of SCFAs related to the positional sensitivity of cetuximab. However, we can conclude that there is a correlation between the efficacy of cetuximab and the function of SCFAs.

\section{Conclusion}

Most ADRs (skin reactions, hypomagnesemia, mucositis, and transfusion-related reactions) of cetuximab are related to the inhibition of EGFRs and the mode of administration. Diarrhea and nausea are also common ADRs of cetuximab. ADRs of the digestive tract are common in patients treated with cetuximab combined with chemotherapy. Gastrointestinal disasters (GDs) are a manifestation of mucositis in the digestive tract. The health of the digestive tract is closely related to the nutritional status and immune status of the human body, which is the basis of tolerance to ADRs, such as skin reactions and infusion reactions. Moreover, the management of the digestive tract to treat diarrhea is a necessary measure in the treatment of hypomagnesemia. The integrity of the IMB, GM-host immune balance, and biological effects of bacterial metabolites (SCFAs) all play a role in intestinal mucositis in CRC patients treated with cetuximab. Gastrointestinal disasters (GDs) are closely related to ADRs in the digestive tract, drug efficacy and the health of the gastrointestinal system affect drug reactivity and efficacy and ultimately the progression of mCRC and prognosis of colorectal patients. Recently, increasing numbers of studies have found that probiotics and prebiotics can restore the balance between GM and the host immune system, reduce ADRs, and improve the antitumor effect $[77,78]$. Probiotics have been proven to be a safe and beneficial choice for IBD and CRC patients [79], with randomized clinical placebocontrolled trials reporting that probiotics did not increase the risk of ADRs, unless the patients with a poor immune system or severely damaged IMB [80]. Therefore, we suggest taking measures to maintain the IMB, regulate the GM-host immune balance, and control intestinal bacterial metabolism in CRC patients treated with cetuximab monotherapy or cetuximab combined with chemotherapy. Probiotics and prebiotics represent promising measures to alleviate ADRs associated with cetuximab, enhance the efficacy of cetuximab, and improve the prognosis of mCRC.

\section{Declarations}

Conflict of interest: The authors declare no conflict of interest.

Acknowledgment: This work was supported by Key $\mathrm{R} \& \mathrm{D}$ and promotion projects in Henan Province: 202102310118, Chinese society of Clinical Oncology fund(CSCO FUND: Y-MX2015-095) and Natural Science Foundation of China (No.81702343).

\section{References}

1. García-Foncillas J, Díaz-Rubio E. Progress in metastatic colorectal cancer: growing role of cetuximab to optimize clinical outcome. Clinical and Translational Oncology, 2010, 12(8): 533-542.

2. Hanck-Silva G, Fatori Trevizan L N, Petrilli R, et al. A Critical Review of Properties and Analytical/Bioanalytical Methods for Characterization of Cetuximab. Critical Reviews in Analytical Chemistry, 2020, 50(2): 125-135.

3. Vincenzi B, Schiavon G, Silletta M, et al. The biological properties of cetuximab. Critical reviews in oncology/ hematology, 2008, 68(2): 93-106.

4. Li X, Fan Z. The epidermal growth factor receptor antibody cetuximab induces autophagy in cancer cells by downregulating HIF- $1 \alpha$ and Bcl-2 and activating the beclin 1/hVps34 complex. Cancer research, 2010, 70(14): 5942-5952.

5. Cunningham D, Humblet Y, Siena S, et al. Cetuximab monotherapy and cetuximab plus irinotecan in irinotecan-refractory metastatic colorectal cancer. New England journal of medicine, 2004, 351(4): 337-345.

6. Ehrenberg R, Halama N. FOLFOX plus cetuximab in firstline therapy of advanced colorectal cancer. Annals of Translational Medicine, 2018, 6(Suppl 2).

7. Van Cutsem E, Köhne C H, Hitre E, et al. Cetuximab and chemotherapy as initial treatment for metastatic colorectal cancer. New England Journal of Medicine, 2009, 360(14): 1408-1417.

8. Tol J, Koopman M, Cats A, et al. Chemotherapy, bevacizumab, and cetuximab in metastatic colorectal cancer. New England Journal of Medicine, 2009, 360(6): 563572.

9. Adkins D, Ley J, Neupane P, et al. Palbociclib and cetuximab in platinum-resistant and in cetuximab-resistant human papillomavirus-unrelated head and neck cancer: a multicentre, multigroup, phase 2 trial. The Lancet Oncology, 2019, 20(9): 1295-1305.

10. Tonini G, Calvieri A, Vincenzi B, et al. First-line targeted therapies in the treatment of metastatic colorectal cancer-role of cetuximab. OncoTargets and therapy, 2009, 2: 73.

11. Tejpar S, Stintzing S, Ciardiello F, et al. Prognostic and predictive relevance of primary tumor location in patients with RAS wild-type metastatic colorectal cancer: retrospective analyses of the CRYSTAL and FIRE-3 trials. JAMA oncology, 2017, 3(2): 194-201. 
12. Laurent-Puig P, Cayre A, Manceau G, et al. Analysis of PTEN, BRAF, and EGFR status in determining benefit from cetuximab therapy in wild-type KRAS metastatic colon cancer. Journal of clinical oncology, 2009, 27(35): 5924-5930.

13. Nagata $Y$, Lan K H, Zhou X, et al. PTEN activation contributes to tumor inhibition by trastuzumab, and loss of PTEN predicts trastuzumab resistance in patients. Cancer cell, 2004, 6(2): 117-127.

14. Lenz H J. Anti-EGFR mechanism of action: antitumor effect and underlying cause of adverse events. Oncology, 2006, 20(5_Suppl_2).

15. Bronte G, Cicero G, Cusenza S, et al. Monoclonal antibodies in gastrointestinal cancers. Expert opinion on biological therapy, 2013, 13(6): 889-900.

16. Wen F, Li Q. Treatment dilemmas of cetuximab combined with chemotherapy for metastatic colorectal cancer. World journal of gastroenterology, 2016, 22(23): 5332.

17. Lenz H J. Cetuximab in the management of colorectal cancer. Biologics: targets \& therapy, 2007, 1(2): 77.

18. Fornasier G, Francescon S, Baldo P. An update of efficacy and safety of cetuximab in metastatic colorectal cancer: a narrative review. Advances in therapy, 2018, 35(10): 1497-1509.

19. Hofheinz R D, Segaert S, Safont M J, et al. Management of adverse events during treatment of gastrointestinal cancers with epidermal growth factor inhibitors. Critical Reviews in Oncology/Hematology, 2017, 114: 102-113.

20. Lacouture M E, Anadkat M J, Bensadoun R J, et al. Clinical practice guidelines for the prevention and treatment of EGFR inhibitor-associated dermatologic toxicities. Supportive Care in Cancer, 2011, 19(8): 1079-1095.

21. Van Cutsem E, Tejpar S, Vanbeckevoort D, et al. Intrapatient cetuximab dose escalation in metastatic colorectal cancer according to the grade of early skin reactions: the randomized EVEREST study. Journal of clinical oncology, 2012.

22. Roselló S, Blasco I, García Fabregat L, et al. Management of infusion reactions to systemic anticancer therapy: ESMO Clinical Practice Guidelines. Annals of Oncology, 2017, 28(suppl_4): iv100-iv118.

23. Gibson R J, Keefe D M K, Lalla R V, et al. Systematic review of agents for the management of gastrointestinal mucositis in cancer patients. Supportive Care in Cancer, 2013, 21(1): 313-326.

24. Abu-Amna M, Bar-Sela G. Increase in cetuximab-induced skin rash and hypomagnesemia in patients receiving concomitant treatment with proton pump inhibitors (PPIs): a possible drug interaction?. Cancer chemotherapy and pharmacology, 2019, 83(3): 545-550.

25. Moutsopoulos N M, Konkel J E. Tissue-specific immunity at the oral mucosal barrier. Trends in immunology, 2018, 39(4): 276-287.

26. Salvatori F, Siciliano S, Maione F, et al. Confocal laser endomicroscopy in the study of colonic mucosa in IBD patients: a review. Gastroenterology research and practice, 2012, 2012.

27. Anbazhagan A N, Priyamvada S, Alrefai W A, et al. Patho- physiology of IBD associated diarrhea. Tissue barriers, 2018, 6(2): e1463897.

28. Maughan T S, Adams R A, Smith C G, et al. Addition of cetuximab to oxaliplatin-based first-line combination chemotherapy for treatment of advanced colorectal cancer: results of the randomised phase 3 MRC COIN trial. The Lancet, 2011, 377(9783): 2103-2114.

29. Sánchez de Medina F, Romero-Calvo I, Mascaraque C, et al. Intestinal inflammation and mucosal barrier function. Inflammatory bowel diseases, 2014, 20(12): 2394-2404.

30. Van Cutsem E, Kohne C H, Láng I, et al. Cetuximab plus irinotecan, fluorouracil, and leucovorin as first-line treatment for metastatic colorectal cancer: updated analysis of overall survival according to tumor KRAS and BRAF mutation status. J Clin Oncol, 2011, 29(15): 20112019.

31. Goffin J R, Zbuk K. Epidermal growth factor receptor: pathway, therapies, and pipeline. Clinical therapeutics, 2013, 35(9): 1282-1303.

32. France M M, Turner J R. The mucosal barrier at a glance. Journal of Cell Science, 2017, 130(2): 307-314.

33. Merga Y, Campbell B J, Rhodes J M. Mucosal barrier, bacteria and inflammatory bowel disease: possibilities for therapy. Digestive diseases, 2014, 32(4): 475-483.

34. Li H, Limenitakis J P, Fuhrer T, et al. The outer mucus layer hosts a distinct intestinal microbial niche. Nature communications, 2015, 6(1): 1-13.

35. YasudaOnozawa Y, Handa O, Naito Y, et al. Rebamipide upregulates mucin secretion of intestinal goblet cells via Akt phosphorylation. Molecular Medicine Reports, 2017, 16(6): 8216-8222.

36. Olivares-Villagómez D, Van Kaer L. Intestinal intraepithelial lymphocytes: sentinels of the mucosal barrier. Trends in immunology, 2018, 39(4): 264-275.

37. Gaudier E, Jarry A, Blottiere H M, et al. Butyrate specifically modulates MUC gene expression in intestinal epithelial goblet cells deprived of glucose. American Journal of Physiology-Gastrointestinal and Liver Physiology, 2004, 287(6): G1168-G1174.

38. Almohazey D, Lo Y H, Vossler C V, et al. The ErbB3 receptor tyrosine kinase negatively regulates Paneth cells by PI3K-dependent suppression of Atoh1. Cell Death \& Differentiation, 2017, 24(5): 855-865.

39. Heukels P, van Hulst J A C, van Nimwegen M, et al. Enhanced Bruton's tyrosine kinase in B-cells and autoreactive IgA in patients with idiopathic pulmonary fibrosis. Respiratory research, 2019, 20(1): 232.

40. Johansson M E V, Larsson J M H, Hansson G C. The two mucus layers of colon are organized by the MUC2 mucin, whereas the outer layer is a legislator of host-microbial interactions. Proceedings of the national academy of sciences, 2011, 108(Supplement 1): 4659-4665.

41. Araujo G, Yunta C, Terré M, et al. Intestinal permeability and incidence of diarrhea in newborn calves. Journal of dairy science, 2015, 98(10): 7309-7317.

42. Coëffier M, Déchelotte P, Ducrotté P. Intestinal permeability in patients with diarrhea-predominant irritable bowel syndrome: is there a place for glutamine supple- 
mentation?. Gastroenterology, 2015, 148(5): 1079-1080.

43. Kuipers EJ, Grady WM, Lieberman D. et al. Colorectal cancer. nature reviews disease primers, 15065 (2015).

44. De Martel C, Ferlay J, Franceschi S, et al. Global burden of cancers attributable to infections in 2008: a review and synthetic analysis. The lancet oncology, 2012, 13(6): 607-615.

45. Grivennikov S I, Greten F R, Karin M. Immunity, inflammation, and cancer. Cell, 2010, 140(6): 883-899.

46. Schmidt A, Weber O F. In memoriam of Rudolf virchow: a historical retrospective including aspects of inflammation, infection and neoplasia[M]//Infection and inflammation: impacts on oncogenesis. Karger Publishers, 2006, 13: 1-15.

47. Liu X, Cheng Y, Shao L, et al. Alterations of the Predominant Fecal Microbiota and Disruption of the Gut Mucosal Barrier in Patients with Early-Stage Colorectal Cancer. BioMed Research International, 2020, 2020.

48. Spencer S P, Fragiadakis G K, Sonnenburg J L. Pursuing human-relevant gut microbiota-immune interactions. Immunity, 2019, 51(2): 225-239.

49. Marchesi J R, Adams D H, Fava F, et al. The gut microbiota and host health: a new clinical frontier. Gut, 2016, 65(2): 330-339.

50. Ratajczak W, Rył A, Mizerski A, et al. Immunomodulatory potential of gut microbiome-derived short-chain fatty acids (SCFAs). Acta Biochimica Polonica, 2019, 66(1): 1-12.

51. Singh N, Thangaraju M, Prasad P D, et al. Blockade of dendritic cell development by bacterial fermentation products butyrate and propionate through a transporter (Slc5a8)-dependent inhibition of histone deacetylases. Journal of Biological Chemistry, 2010, 285(36): 2760127608.

52. Ng K M, Aranda-Díaz A, Tropini C, et al. Recovery of the gut microbiota after antibiotics depends on host diet, community context, and environmental reservoirs. Cell host \& microbe, 2019, 26(5): 650-665. e4.

53. Sotelo M J, García-Paredes B, Aguado C, et al. Role of cetuximab in first-line treatment of metastatic colorectal cancer. World journal of gastroenterology: WJG, 2014, 20(15): 4208.

54. Nosho K, Baba Y, Tanaka N, et al. Tumour-infiltrating Tcell subsets, molecular changes in colorectal cancer, and prognosis: cohort study and literature review. The Journal of pathology, 2010, 222(4): 350-366.

55. Maeda $\mathrm{H}$, Akaike T. Nitric oxide and oxygen radicals in infection, inflammation, and cancer. BIOCHEMISTRY C/C OF BIOKHIMIIA, 1998, 63: 854-865.

56. Martens E C, Neumann M, Desai M S. Interactions of commensal and pathogenic microorganisms with the intestinal mucosal barrier. Nature Reviews Microbiology, 2018, 16(8): 457-470.

57. Tan J, McKenzie C, Potamitis M, et al. The role of shortchain fatty acids in health and disease[M]//Advances in immunology. Academic Press, 2014, 121: 91-119.

58. Panebianco C, Andriulli A, Pazienza V. Pharmacomicrobiomics: exploiting the drug-microbiota interactions in anticancer therapies. Microbiome, 2018, 6(1): 92.

59. Adak A, Khan M R. An insight into gut microbiota and its functionalities. Cellular and Molecular Life Sciences, 2019, 76(3): 473-493.

60. El Akkad I, Hobson P N. Effect of antibiotics on some rumen and intestinal bacteria. Nature, 1966, 209(5027): 1046-1047.

61. Meunier A, Nerich V, Fagnoni-Legat C, et al. Enhanced emergence of antibiotic-resistant pathogenic bacteria after in vitro induction with cancer chemotherapy drugs. Journal of Antimicrobial Chemotherapy, 2019, 74(6): 1572-1577.

62. Balkwill F, Mantovani A. Inflammation and cancer: back to Virchow?. The lancet, 2001, 357(9255): 539-545.

63. De Almeida C V, de Camargo M R, Russo E, et al. Role of diet and gut microbiota on colorectal cancer immunomodulation. World journal of gastroenterology, 2019, 25(2): 151.

64. Le Poul E, Loison C, Struyf S, et al. Functional characterization of human receptors for short chain fatty acids and their role in polymorphonuclear cell activation. Journal of Biological Chemistry, 2003, 278(28): 2548125489.

65. Park J, Kim M, Kang S G, et al. Short-chain fatty acids induce both effector and regulatory $\mathrm{T}$ cells by suppression of histone deacetylases and regulation of the mTOR-S6K pathway. Mucosal immunology, 2015, 8(1): 80-93.

66. Johnstone R W. Histone-deacetylase inhibitors: novel drugs for the treatment of cancer. Nature reviews Drug discovery, 2002, 1(4): 287-299.

67. Chang P V, Hao L, Offermanns S, et al. The microbial metabolite butyrate regulates intestinal macrophage function via histone deacetylase inhibition. Proceedings of the National Academy of Sciences, 2014, 111(6): 22472252.

68. Singh N, Gurav A, Sivaprakasam S, et al. Activation of Gpr109a, receptor for niacin and the commensal metabolite butyrate, suppresses colonic inflammation and carcinogenesis. Immunity, 2014, 40(1): 128-139.

69. Brown A J, Goldsworthy S M, Barnes A A, et al. The Orphan G protein-coupled receptors GPR41 and GPR43 are activated by propionate and other short chain carboxylic acids. Journal of Biological Chemistry, 2003, 278(13): 11312-11319.

70. Cao D D, Xu H L, Xu X M, et al. The impact of primary tumor location on efficacy of cetuximab in metastatic colorectal cancer patients with different Kras status: a systematic review and meta-analysis. Oncotarget, 2017, 8(32): 53631.

71. Holch J W, Ricard I, Stintzing S, et al. The relevance of primary tumour location in patients with metastatic colorectal cancer: a meta-analysis of first-line clinical trials. European Journal of Cancer, 2017, 70: 87-98.

72. Brule S Y, Jonker D J, Karapetis C S, et al. Location of colon cancer (right-sided versus left-sided) as a prognostic factor and a predictor of benefit from cetuximab in NCIC CO. 17. European journal of cancer, 2015, 51(11): 14051414. 
73. Lu H J, Lin J K, Chen W S, et al. Primary tumor location is an important predictive factor for wild-type KRAS metastatic colon cancer treated with cetuximab as front-line bio-therapy. Asia-Pacific Journal of Clinical Oncology, 2016, 12(3): 207-215.

74. Koh A, De Vadder F, Kovatcheva-Datchary P, et al. From dietary fiber to host physiology: short-chain fatty acids as key bacterial metabolites. Cell, 2016, 165(6): 13321345.

75. Cummings J H, Pomare E W, Branch W J, et al. Short chain fatty acids in human large intestine, portal, hepatic and venous blood. Gut, 1987, 28(10): 1221-1227.

76. Hu J, Lin S, Zheng B, et al. Short-chain fatty acids in control of energy metabolism. Critical reviews in food science and nutrition, 2018, 58(8): 1243-1249.
77. Agatis J, Campus D. Application of probiotic, prebiotic and synbiotic for the control of streptococcosis in tilapia Oreochromis niloticus. Pakistan Journal of Biological Sciences, 2015, 18(2): 59-66.

78. Mego M, Holec V, Drgona L, et al. Probiotic bacteria in cancer patients undergoing chemotherapy and radiation therapy. Complementary Therapies in Medicine, 2013, 21(6): 712-723.

79. Pandey K R, Naik S R, Vakil B V. Probiotics, prebiotics and synbiotics-a review. Journal of food science and technology, 2015, 52(12): 7577-7587.

80. Boyle R J, Robins-Browne R M, Tang M L K. Probiotic use in clinical practice: what are the risks?. The American journal of clinical nutrition, 2006, 83(6): 1256-1264.

Cite this article as: $\mathrm{Wu} \mathrm{Y,} \mathrm{Jia} \mathrm{H}$, Bao X, et al. Gastrointestinal disasters of cetuximab in the treatment of metastatic colorectal cancer: Mechanism and its effect on prognosis[J]. Aging Pathobiology and Therapeutics, 2020, 2(2): 64-72. 